\title{
Interdisciplinary transforming of the functions of pedagogical comparativistics
}

\author{
Natalia Naydenova ${ }^{1, *}$, Irina Tagunova ${ }^{1}$, and Igor Sukhin ${ }^{1}$ \\ ${ }^{1}$ Institute for the Education Development Strategy of Russian Education Academy, 105062, 5/16 \\ Makarenko str., Moscow, Russia
}

\begin{abstract}
The article presents a classification of interdisciplinary transformations of the functions of comparative pedagogy in the information age. The problem of transforming comparative pedagogy into pedagogical comparativistics was studied. The key functions of pedagogical comparativistics are revealed. Classification is considered in the evolutionary key. In the classification of the transformation of key functions, four types of evolutionary transformations were defined: 1) divergent, 2) parallel, 3) co-evolutionary, 4) convergent. Thus, the authors pointed to hybrid transformations under the influence of other sciences: cognitive neuroscience, mathematics, psychology, statistics, evaluation of results in social sciences, informatics and others. The main function was recognized as a comparative function that combines together analytical, representative, instrumental, evaluation and prognostic functions into a single whole.
\end{abstract}

\section{Comparative education}

Education takes on a transnational character. Increasingly, public education systems interact with transnational actors, for example, with the OECD and the EU. International competition in the knowledge economy and in cultural development affects education, especially in continuing education. Thus, the study of the experience of the development of national education systems is of vital social importance [1-4]. Enhanced requirements for the improvement or remodeling of education arise particularly under the influence of rating estimates in international comparative studies [5-7]. Russia is no exception. Similar ways of identifying and combating education problems also arise in other national contexts.

The comparative paradigm in education is substantially transformed in the information age, taking into account the status of international comparisons and the influence of the transnational expertise of current discourses in education. Therefore, comparativeness becomes particularly important. Since comparativistics is an interdisciplinary domain of science, the concept of comparative pedagogy, which is common in the post-Soviet space, in our opinion, is to speak of pedagogical comparativism as a separate area within general comparativistics, and on the other hand, pedagogical comparativistics remains a separate field within general pedagogy. That is, there is a hybrid transformation of concepts:

\footnotetext{
* Corresponding author: naydenova@my.com
} 
monodiscipline within the interdisciplinary sphere, and also - an interdisciplinary discipline within monodiscipline [8-10].

Nevertheless, there is an urgent need to analyze and critically study educational comparativism. The comparative paradigm in education is a reformed intersection between science and society, therefore the development of education in a comparative perspective is transformed as the education science (in the world educational space it is comparative education), as well as science in the social sciences. Consequently, issues related to changing the functions of pedagogical comparative studies should be considered at the interdisciplinary level. As the great Gandhi said (see the epigraph), in order to understand the time it is necessary to change with him. Consequently, the functions of comparative education change in time. To this end, it is necessary to determine the key functions of pedagogical comparativistics in modern times.

\section{Key functions of pedagogical comparativistics}

Education takes on a transnational character. Increasingly, public education systems interact Among the main functions of comparative pedagogy are the following: 1) cognitive, 2) instrumental, 3) explanatory, 4) managerial, 5) prognostic [8-10]. Although the key function by definition is a comparative function. There can be no comparative pedagogy, comparative education or pedagogical comparativistics (we will regard these concepts as synonymous: comparative pedagogy in the post-Soviet space, comparative education in most developed countries, pedagogical comparativistics in the scientific community of comparativists of different countries in the information age) without comparing different aspects education systems of different countries.

According to Harold Noah (1985) and Farooq Joubish (2009), comparative education has four functions [11-12]:

1. Descriptive (study of educational systems, processes or results).

2. Developing (assistance in the development of educational institutions and practical applications).

3. Communicative (emphasize the relationship and interaction between education and society).

4. Generalizing (the creation of generalized recommendations on the effectiveness of education, which operate in more than one country).

Comparing the education systems of two or more different countries is a key function of comparative education, and sometimes it is mistakenly believed that this only applies to mass comparative studies. In fact, from the earliest studies, comparativists often avoided such approaches, preferring rather to focus on comparing education in one country in a temporal key (a comparative-retrospective analysis in which the main criterion is time), that is, a historico-comparative function is used. Nevertheless, some large-scale projects, such as the PISA and TIMSS studies, have drawn important conclusions based on an explicitly comparative macroanalysis of massive data sets.

Today, the cognitive function, which includes the following activities, remains a key function: a) studying the specificity of education in different countries; b) identification of ways of transformation in the education systems of different countries in accordance with global challenges; c) analysis of concepts, approaches, methods, methods and tools of certain models and theories of modernization of education; d) standards for organizing, conducting and analyzing the results of comparative theoretical and empirical research in the field of education.

The instrumental function of pedagogical comparativistics is the development of a research tool for searching, measuring and evaluating, processing, analyzing, summarizing and comparing primary comparative information. 
The explanatory function, first of all, is aimed at interpreting the obtained research results.

The administrative function of pedagogical comparativistics, in particular, is expressed in: a) the development of recommendations, proposals, evaluation of various characteristics of the object under study; b) the development of comparative indicators.

The prognostic function is called upon to reveal the tendencies of the development of this or that phenomenon in education, the foreseeing of the possible state of the phenomenon in a specific forecast period.

The existence of different types of classification of the functions of comparative pedagogy in modern pedagogy leads to different interpretations of these functions, although often the same functions are simply denoted by different words, and the content of the function remains the same. Globalization was sharply reflected in comparative pedagogy, and, consequently, it led to the transformation of functions in a meaningful aspect.

\section{Transforming the functions of comparative pedagogy}

Changes in the functions of comparative pedagogy occur continuously and in different countries along different paths. But in any case, such a process of transformation has an evolutionary character. Just as there is an evolution of the development of comparative pedagogy as merely a field of general pedagogy in pedagogical comparativistics as an interdisciplinary science.

Thus, evolutionary changes are of several types: 1) divergent, 2) parallel, 3) coevolutionary, 4) convergent [13].

Divergent transformations of functions are characterized by the fact that similar functions in terms of their content characteristics are very similar. But in different countries have different functional. That is, there is a division of a single function according to its content content into different subfunctions. For example, an explanatory function requires verification comparativists conclusions: 1) The findings are supported by references to the findings of other comparativists without the use of evidence-based methods only based on a review of scientific literature; 2) the conclusions are supported by references to other comparativists, but with a discourse on the reliability and validity of the conclusions; 3 ) the conclusions are supported by the results of various comparative studies; 4) the conclusions are proved with the help of special methods from other sciences.

Thus, the divergent evolution of the transformation of comparative education functions is influenced by modern conditions of development of the social sciences, including the related sciences, such as mathematics, linguistics, computer science, and others. Such a transformation is called selective divergence, as the findings and conclusions, which make different comparativists, are explained differently depending on the selective set of arguments. Sometimes within one country there is such a selective divergence of the functions of pedagogical comparativistics. And at least in any country there are two divergence approaches: 1 ) traditional (only a review and belief in the conclusions of foreign comparativists); 2) global (overview comparativists from different countries according to the explanation of the changes in the studied country and proof of the representativeness of the selection of works and comparativists, countries used their fundamentals looks verification methods).

Parallel evolution is characterized by the fact that, in spite of the separation of content functions in different countries, the functions are transformed into the modern age the same, but the final change in a particular country, for all the parallel development are clear differences between the countries. For example, the transformation of the predictive function is clearly parallel evolution: for example, when comparing the results of comparative studies in developed countries are based on the findings of a representative 
national and international studies at a certain comparability of comparison tools. In other countries, forecasts are formed only on the basis of the conclusions of a particular group of comparativists, or even one person, that is, the prognostic function becomes interpretational-prognostic.

The co-evolutionary form of transformation is characterized by similar changes, but the final transformation, with obvious differences, is very similar in content. For example, the transformation of the cognitive function has the same character in different countries, but the priorities for changes are placed in different ways: almost everywhere in the first place is the study of the development of education in foreign countries.

And, for example, the definition of ways of transformation in education systems of different countries in accordance with global challenges may be absent in the content of the cognitive function.

The most important attention is paid to the convergent type of transformation of functions: mixing of the contents of different functions occurs, as indicated above, not just a predictive function, but an interpretation-prognostic function. The historical-comparative function has become comparative, and in the information age it is already instrumentalcomparative. Instrumental function converges to the reference function into a single function: a comparison and comparison tool is closely related functions, that is, there is a convergence of functions, especially in the context of globalization and intense computerization of research [14].

\section{Conclusion}

In neuroscience, the study of thought processes and the effectiveness of these thought processes for achieving specific goals can be informative, but attracting the main function of pedagogical comparativistics - comparative - allows to get more information to these studies, because now you can compare your results with reliable statistical significance.

For example, in addition to determining the neural-scientific characteristics, it becomes possible to compare people with certain mental health problems with results on the general population, which will help to find out the possible causes of identified cognitive abnormalities that are above or below the average level. Comparing pedagogy, strategy, practice and learning outcomes in one country with other nations, you receive information. For example, when comparing the educational system of education in Kazakhstan and Russia, one should begin by comparing the student's progress in schools. Then you can compare the training of teachers and even the payment of teachers. After all, attitudes toward learning and teachers in these countries can be different because of the difference in cultural, national and religious codes of educational development. In this case, you do not need to visit the country to make comparisons [15].

An additional function of pedagogical comparativistics should be considered regional: a comparison within one country on a regional basis. This is especially important for large countries, where training in certain regions can differ in their components. In the United States, you can compare education in large cities with suburban school districts, private schools with public schools. Ultimately, although, like many social scientists, comparativists from pedagogy hope to answer questions about effective education. They want to know what methods give universal results, and they hope to find out why this practice produces different results, depending on where and who engages in education. The comparators can map education systems according to the belonging of educational institutions to a particular economic class.

After all, as research becomes more rigorous in the field of representation, the development of tools in accordance with the training program and the analysis of results, involving the broader layers of students, training and administrating subjects of education 
of each country participating in the comparative study. Therefore, comparativists can answer questions about the nature of teaching and learning, its universals, and also under what conditions are optimal or not optimal one or another method. Thus, a new function is introduced-representative.

Consequently, in the information age there is an intensive rapid change in the information field of comparative research, which inevitably leads to an interdisciplinary transformation of the basic, key, complementary and other functions of pedagogical comparativistics.

So, the comparative function acquires an interdisciplinary hybrid character: the cognitive context of comparisons, the demographic and socio-economic perspectives, the representativeness of comparisons, the measurable assessment and other purely pedagogical comparisons [16].

Thus, pedagogical comparativistics contributes to the development of a comparative pedagogical culture among all subjects of the educational process. Therefore, pedagogical comparativistics as a teaching discipline sets as its main task the formation of a mass teacher-comparative literacy, which is a necessary condition for the formation of civil society.

In the information age, it is necessary to emphasize once again the key functions that make it possible to raise the level of the comparative culture of society. There are:

- cognitive - a certain way of knowing the teacher-comparative constructed educational reality and revealing the laws of its development;

- analytical - assessment of the state of educational systems at the local, regional, national and supranational levels in different countries and educational spaces and the performance of various comparativists in the pedagogical process of comparing the effectiveness of modernizing education in accordance with international requirements in different countries; - prognostic - the development of scientifically based forecasts about the trends (prospects) for the development of comparative studies in the field of interdisciplinary education;

- management - using the results of comparative research in the field of education for the development and adoption of management decisions;

- instrumental - improvement of research methods of the constructed educational reality;

- teacher-comparative socialization - the preparation and integration (entry) of the individual, social groups into the pedagogical life of society, provided competent comparisons with the key trends in the development of education in the modern transforming global world;

- ideological - the use of comparative research in the propagation of their ideas and criticism of others.

Pedagogical comparative studies create a certain theoretical and scientificmethodological base necessary for the development of comparative education itself and for the improvement of pedagogical consciousness in the whole society. Scientific knowledge in the field of pedagogical comparativistics makes it possible to predict and construct an educational reality, to track positive and negative trends in the development of the processes of comparing education within and between countries and, if necessary, to make the necessary methodological adjustments in the current conditions of globalization.

Pedagogical comparativistics as a science can also perform certain educational and public functions: to formulate certain models of sustainable development, educational needs at different levels of education, the values of the educational culture and thereby consolidate society for achieving any goals (for example, building a modern state for sustainable development). 
The article was prepared within the framework of the project No. 17-06-00122a for the RFBR.

\section{References}

1. M. Bray, M. Martin (Eds.), Tertiary education in small states: planning in the context of globalization (UNESCO/IIEP, Paris, 2011)

2. M. Crossley, Journal of International and Comparative Education, 1 (2012)

3. C. Brock, N. Alexiadou, Education Around the World: a comparative introduction. (Bloomsbury, London and New York, 2013)

4. P. Morris, Journal of Comparative and International Education, 45, 3 (2015)

5. J. Kauko, W. Wermke, Comparative Education Review 62, 2 (2018)

6. B. Uttl, et al., Meta-analysis of faculty's teaching effectiveness (2016)

7. W. Wieland, G. Höstfält, Education Inquiry, 5, 4 (2014)

8. N. N. Naydenova, Otechestvennaya I Zarubezhnaya Pedagogika, 1 (2012)

9. A. L. Moore, Comparative Literature and Culture, 15, 6 (2013)

10. J. Moran, Interdisciplinarity (Routledge, London, 2002)

11. M.A. Eckstein, H.J. Noah, Quarterly Review of Education, 15 (1985)

12. F. Joubish, Educational research: from deign to report writing, department of education, federal Urdu university (Karachi, 2009)

13. B. J. Alters, S.M. Alters defending evolution: a guide to the creation (Jones and Bartlett, MA, 2001)

14. Steller B., Kuhn C., Welcome to Germany 2.0: Move to where you are welcome? (2013)

15. N. N. Naydenova, Pedagogical Journal of Bashkortostan, 61, 6 (2015)

16. Smeyers, P., Bridges, D., Burbules, N. C., \& Griffiths, M. (Eds.). International handbook of interpretation in educational research (Springer, Dordrecht, 2015) 